OPEN ACCESS

Edited by:

Tifei Yuan,

Shanghai Mental Health Center

(SMHC), China

Reviewed by:

Yansong $\mathrm{Li}$,

Nanjing University, China

Fangang Meng,

Capital Medical University, China

*Correspondence:

Tianming Yang

tyang@ion.ac.cn

Shikun Zhan

shikun.zhan@icloud.com

tThese authors have contributed equally to this work.

¥These authors have contributed equally to this work.

Specialty section:

This article was submitted to

Cognition,

a section of the journal

Frontiers in Psychology

Received: 14 May 2017 Accepted: 29 September 2017

Published: 17 October 2017

Citation:

Zhang $C$, Chen Y, Tian S, Wang T, Xie $Y$, Jin $H$, Lin $G$, Gong $H$, Zeljic $K$,

Sun B, Yang T and Zhan S (2017)

Effects of Anterior Capsulotomy on

Decision Making in Patients with

Refractory Obsessive-Compulsive

Disorder. Front. Psychol. 8:1814.

doi: 10.3389/fpsyg.2017.01814

\title{
Effects of Anterior Capsulotomy on Decision Making in Patients with Refractory Obsessive-Compulsive Disorder
}

\section{Chencheng Zhang ${ }^{1+}$, Yilin Chen $2,3+$, Shuaiwei Tian', Tao Wang ${ }^{1}$, Yile Xie ${ }^{4}$, Haiyan Jin ${ }^{5}$, Guozhen Lin ${ }^{5}$, Hengfen Gong ${ }^{6}$, Kristina Zeljic ${ }^{2}$, Bomin Sun ${ }^{1}$, Tianming Yang ${ }^{2 *}$ and Shikun Zhan ${ }^{1 * \neq}$}

\begin{abstract}
' Department of Functional Neurosurgery, Ruijin Hospital, Shanghai Jiao Tong University School of Medicine, Shanghai, China, ${ }^{2}$ Institute of Neuroscience, Key Laboratory of Primate Neurobiology, CAS Center for Excellence in Brain Science and Intelligence Technology, Chinese Academy of Sciences, Shanghai, China, ${ }^{3}$ University of Chinese Academy of Sciences, Beijing, China, ${ }^{4}$ Shanghai Jiao Tong University School of Medicine, Shanghai, China, ${ }^{5}$ Department of Psychiatry, Ruijin Hospital, Shanghai Jiao Tong University School of Medicine, Shanghai, China, ${ }^{6}$ Department of Psychiatry, Pudong District Mental Health Center, Shanghai, China
\end{abstract}

Despite various lines of evidence implicating impaired decision-making ability in individuals with obsessive-compulsive disorder (OCD), neuropsychological investigation has generated inconsistent findings. Although the cortico-striato-thalamo-cortical (CSTC) circuitry has been suggested, the involvement of the cortex has not yet been fully demonstrated. Moreover, it is unknown whether surgical intervention on the CSTC circuitry results in a predicted improvement of decision-making ability of OCD. Here we present a study of decision making based on the lowa Gambling Task (IGT) to investigate decision making in a large sample of individuals with treatment-resistant $O C D$ with and without anterior capsulotomy (AC). Task performance was evaluated in healthy subjects, individuals with $O C D$ that had not undergone surgery, and postsurgical $O C D$ patients with AC. The latter group was further divided into a short-term postsurgical group and a long-term postsurgical group. We found that the OCD patients without surgery performed significantly worse than the healthy controls on the IGT. There were no significant differences in decision-making between the presurgical OCD patients and those at the short-term postsurgical follow-up. Decision-making ability of the long-term postsurgical OCD patients was improved to the level comparable to that of healthy controls. All clinical symptoms (OCD, depression, and anxiety) assessed by psychiatric rating scales were significantly alleviated post-surgically, but exhibited no correlation with their IGT task performance. Our findings provide strong evidence that OCD is linked to impairments in decision-making ability; that impaired CSTC circuitry function is directly involved in the manifestation of OCD; and that AC related improvements in cognitive functions are caused by long-term plasticity in the brain circuitry.

\footnotetext{
Keywords: obsessive-compulsive disorder, anterior capsulotomy, decision-making, lowa gambling task
} 


\section{INTRODUCTION}

Obsessive-compulsive disorder (OCD) is a symptomatologically heterogeneous psychiatric disorder with a worldwide lifetime prevalence ranging from 2 to $3 \%$ that often leads to chronic disability (Abramowitz et al., 2009). The primary clinical manifestations of this disorder include recurrent, ruminative and anxiety-inducing thoughts (obsessions) and/or repetitively ritualized mental acts or behaviors (compulsions). Existing treatment methods entailing pharmacotherapy and cognitive behavioral therapy (CBT) generally only result in partial symptom improvements, with roughly one third of cases continuing to exhibit severe chronic illness that is resistant to treatment (Bloch et al., 2006, 2012; Mallet et al., 2008; Greenberg et al., 2010; Grant, 2014; Odlaug et al., 2014). Neurosurgical intervention has demonstrated substantial symptom alleviation in such cases (Jung et al., 2014). It typically involves either an ablative procedure or reversible neuromodulation using deep brain stimulation (DBS) at locations within the cortico-striatothalamo-cortical (CSTC) circuitry, from which effects spread throughout the brain (Greenberg et al., 2010; Jung et al., 2014; Pepper et al., 2015).

The CSTC model of OCD is currently the prevailing model in terms of the neural and pathophysiological underpinnings of the disorder (Pauls et al., 2014), and is supported by decades of animal research that have led to findings of a strong association between OCD-like behavior and aberrant functioning of frontostriatal neural circuitry (Fineberg et al., 2011). The functionally distinct loops comprising this circuitry originate in specific territories of the frontal cortex, project to target areas in the striatum and pass through the basal ganglia to the thalamus, with recurrent projections back to their corresponding frontal cortical regions of origin (Milad and Rauch, 2012; Nakao et al., 2014). Research in humans has been heavily informed by findings in animals, and brain imaging studies have been extensively employed in the investigation and refinement of the CSTC model (Whiteside et al., 2004; Norman et al., 2016). Although this area of research has yielded variable results in terms of specific regions and the direction of dysfunction (increased vs. decreased activity) in different studies, abnormal activity in the frontostriatal regions is consistently reported. Resting-state functional magnetic resonance imaging (fMRI) studies most often find increased activity in the orbitofrontal cortex (OFC) and striatum, while symptom provocation paradigms most often find increases in the OFC, caudate and anterior cingulate cortex (ACC) (Saxena et al., 2001; Schlösser et al., 2010; Nakao et al., 2014).

Given the aberrant functioning of the OFC in OCD, it is unsurprising that diminished performance on decision-making tasks is commonly reported in affected individuals. Decision-making ability is a multifaceted higher order function with significant real-world implications that relies on intact functioning of the prefrontal cortex for representing and integrating relevant information. The Iowa Gambling Task (IGT), an extensively used decision-making task paradigm, requires the participant to track the stimulus-reward association by distinguishing between the stimuli with an immediate large reward but a long-term negative consequence and the stimuli with an immediate small reward but a long-term positive consequence. Compared to similar neuropsychological tasks, such as the Wisconsin Card Sorting Test (WCST), the IGT is sensitive to damage and/or dysfunction across a wider range of brain areas, including anterior and posterior regions of the orbitofrontal and ventromedial prefrontal cortex, as well as the dorsolateral prefrontal cortex (Bechara et al., 1998, 2001).

Although a number of studies have investigated the relationship between $\mathrm{OCD}$ and decision-making ability using IGT, their findings have not been consistent. Cavedini et al. (2002) demonstrated that subjects with OCD performed more poorly on the IGT than subjects with panic disorder and healthy controls (Cavedini et al., 2002). Furthermore, they found poor neuropsychological task performance to be a predictor of poor outcome to pharmacological treatments. Cavallaro et al. (2003) also found that OCD patients performed significantly worse on the IGT than both control subjects and schizophrenia patients (Cavallaro et al., 2003). However, Nielen et al. (2002) and Lawrence et al. (2006) found no differences between the performance of OCD patients and control subjects in the IGT (Nielen et al., 2002; Lawrence et al., 2006). Interestingly, Zhang et al. (2015) recently found that medicated, non-medicated and remitted OCD patients all performed significantly worse on the IGT than comparison subjects (Zhang et al., 2015). These inconsistencies may in part be due to the heterogeneity of OCD, the methodological differences, and the sampling constraints in some of the studies.

These inconsistent results indicate that the links between OCD, decision-making and altered prefrontal functions remain unclear. Therefore, studies investigating the effects of surgical intervention in the brain circuitry on OCD and decisionmaking could shed light on the puzzle (Brown et al., 2016). Surprisingly, there has been only one study to date investigating the effects of surgical intervention in OCD on decisionmaking as assessed by the IGT (Csigo et al., 2010). Csigo and colleagues monitored five refractory OCD patients that underwent anterior capsulotomy (AC), and five non-operated refractory OCD patients over a 2-year period. Their results indicated improvements in IGT performance in the former group at the 1- and 2-year follow-ups (but not at the 1-month or 6-month follow-ups) with no improvements in the nonoperated group. Although these results suggest that AC may lead to improved decision-making in OCD, the study was done with a very limited sample size. It is important to understand the difference between short-term and long-term AC effects on decision making, because it would help us to interpret the circuitry affected by AC.

We therefore aimed to conduct a broad and reliable assessment of decision making under risk as measured by the IGT before and after $\mathrm{AC}$ at different time points in a large sample of OCD patients as compared to healthy individuals, while also examining the relationship between task performance and scores on a range of clinical measures. We found that significant improvements in the IGT performance after AC happened only in the patient group that had received the surgery for more than 1 year, suggesting a long-term plasticity 
in the brain circuitry was responsible for such improvements. Furthermore, the improvements in IGT performance could not be explained by their psychiatrical rating scale scores, which showed improvements shortly after AC.

\section{MATERIALS AND METHODS}

\section{Participants}

The sample consisted of 138 subjects divided into four groups: 31 healthy subjects (HCs; mean age $=37.77, S D=10.83$; mean education $=13.30$ years, $S D=4.61$; Female: 14$) ; 51$ pre-operative OCD patients (preO; mean age $=30.71, S D=7.62$; mean education $=13.07$ years, $S D=2.58$ years; mean illness duration $=10.66$ years, $S D=7.12$ years; Female: 15$)$; 24 postoperative short term OCD patients (postST), 3.00 to 5.25 months (average 4 months) after surgery (mean age $=29.29, S D=5.72$; mean education $=13.40$ years, $S D=1.98$ years; mean illness duration $=8.92$ years, $S D=3.55$ years; Female: 8 ); and 32 postoperative long term OCD patients (postLT), 2.00 to 5.00 years (average 3.00 years) after surgery (mean age $=33.41, S D=7.86$; mean education $=13.3$ years, $S D=2.47$ years; mean illness duration $=10.50$ years, $S D=4.85$ years; Female: 12$)$ (Table 1$)$. The patients were recruited via the Department of Psychiatry and Department of Functional Neurosurgery at Ruijin Hospital affiliated to Shanghai Jiao Tong University School of Medicine. The three patient groups were recruited independently. The post-operation groups comprised patients visiting the hospital for the post-surgical follow-up. Measurement of illness duration includes the time period until the post-operative follow-up. The Department of Neurosurgery at Ruijin hospital has a well-established long-term treatment program for refractory OCD (Liu et al., 2008; Zuo et al., 2013; Zhan et al., 2014). All study procedures were performed in accordance with the relevant guidelines and regulations (Nuttin et al., 2014). The diagnosis, symptom severity, and refractory disease status of each referred patient was independently confirmed by three licensed psychiatric professionals according to the Mini-International Neuropsychiatric Interview for DSM-IV-TR (M.I.N.I. 6.0.0) after a complete review of their medical history (Sheehan et al., 1998). Patients with a concurrent diagnosis of bipolar disorder or a history of manic/hypomanic episodes, psychotic symptoms, substance abuse, neurological illness or brain injury/trauma were excluded from the study.

Education-level matched control subjects were recruited via an advertisement at Ruijin Hospital. Exclusion criteria included a lifetime occurrence of a DSM-IV-TR axis I disorder or neurological disease and the current use of any medication that affects cognitive functions.

All subjects provided written informed consent for participation and publication; the study was approved by the Ethics Committee of Ruijin Hospital affiliated with Shanghai Jiao Tong University School of Medicine in accordance with the Declaration of Helsinki. A portion of this patient sample were participants in a parallel study investigating the safety and effectiveness of capsulotomy in refractory OCD (clinical.trails.gov: NCT02375152).

\section{Neuropsychological and Clinical Assessment}

We used the computerized version of the IGT to examine decision making under ambiguity (Bechara et al., 2000). The IGT involves ascertaining the profile of wins and losses associated with four card decks (A, B, C, and D). Cards can be grouped into two advantageous $(C$ and $D)$ and two disadvantageous (A and $\mathrm{B}$ ) alternatives. The two disadvantageous decks (A and B) are considered 'risky', as they yield large immediate wins, but intermittently result in even larger losses and ultimately lead to debt. The other two decks (C and D) deliver smaller wins with small intermittent losses, resulting in overall gain. Each trial involves the selection of one card from four card decks, with a total of 100 trials. To subjects, the outcome probabilities are initially unspecified, the total number of trials is unknown, and the objective is to win as much virtual money as possible. As the task progresses, the contingencies can be inferred based on the feedback from the previous outcomes. The test reflects decision-making ability under uncertain and ambiguous conditions that require the use of reward feedback. We adopted a cross sectional experimental design to eliminate the influence of learning effects on task performance, as reordering the decks between testing sessions based on reward properties is not sufficient to control for these effects if the basic rule underlying this particular task has been learned.

The three OCD patient groups were clinically evaluated using three psychiatric rating scales: The Yale-Brown ObsessiveCompulsive Scale (Y-BOCS) (Goodman et al., 1989), the Hamilton Depression Scale (HAMD) (Hamilton, 1986), and the Hamilton Anxiety Scale (HAMA) (Maier et al., 1988). Mean and standard deviation values for each group are shown in Table 2.

\section{Surgical Procedure of Anterior Capsulotomy}

Before the surgery, patients received MRI scans for determining the target coordinates (1.5 T; General Electric, Madison, WI, United States). During the surgery, a stereotaxic surgical frame (Elekta Inc, Stockholm, Sweden) was fixed to the skull under local anesthesia to ensure precise targeting. A localization box was temporarily fixed to the frame. Data were fed into a high-performance computer and the target was identified based on stereotactic MRI visualization of the internal capsule. Coordinates for the bilateral anterior capsule target were 22-25 $\mathrm{mm}$ anterior to the middle of the anterior commissure (AC)-posterior commissure (PC) line, horizontal to the AC - PC, and 18-20 mm lateral to the midline. Monopolar electrodes (Radionoics, Burlington, MA, United States) were subsequently led into the target brain points to a position previously detailed through a drilled skull hole. The position of the electrode was set by a micromanipulator placed on the frame. Neurological testing was carried out to ensure that there were no impairments of motor or sensory functions. Brain lesions were then made with radio-frequency-stimulation below and right of the target points, first on the right side, then on the left side. These radiofrequency lesions were created at $80^{\circ} \mathrm{C}$ for $60 \mathrm{~s}$. Further 
details of the surgical procedure can be found in the previous reports (Liu et al., 2008; Zhan et al., 2014).

\section{Statistical Analysis}

\section{Patient Background and Psychiatric Rating Scales}

Patients with OCD were divided into three groups according to their surgical status: (1) the pre-operative group, (2) the post-operative short-term group, and (3) the post-operative long-term group. Descriptive variables including age and education duration, together with clinical variables including illness duration and scores on clinical measures were first evaluated using the Brown-Forsythe variance test for equal variances. As the participant groups showed significant different variances in age $[F(3,134)=6.00, p=0.0007]$, education duration $[F(3,134)=9.15, p=0.000015]$, illness duration $[F(2,104)=3.92, p=0.023]$, YBOCS $[F(2,104)=24.79$, $p=0]$, HAMD $[F(2,104)=20.43, p=0]$, and HAMA $[F(2,104)=27.35, p=0]$, Welch tests were applied to the rest of the analyses for testing the differences in the mean. Rank-sum tests were then applied to compare the data in pairs.

\section{IGT Performance}

The task consisted of 100 trials, which were divided into five blocks of 20 trials for the purpose of analysis. For each block, the net score was calculated as the difference between the numbers of advantageous and disadvantageous choices. A mixed ANOVA was then applied to examine the net score differences within and between groups. To increase statistical power, we also calculated the net scores by combining the trials from the blocks 2 and 3 , and the blocks 4 and 5 to further investigate the learning process in the post hoc analysis. Differences between groups were tested within these testing periods (Block 1, Block $2+3$, and Block $4+5)$. Correlation analyses between the net scores and the descriptive/clinical variables were then carried out.

\section{Strategy Analyses}

A mixed between-within $4^{*} 4$ ANOVA was applied to analyze the main differences in the strategy and participant group factors. Strategy was the within-subject factor, which included the stay probabilities after: wins based on the choices from the good decks, losses based on the choices from the good decks, wins based on the choices from the bad decks, and losses based on the choices from the bad decks. Participant group was the betweengroup factor, which included the healthy control group, the pre-operation OCD group, the post-operation short-term OCD patient group and the post-operation long-term OCD patient group.

\section{RESULTS}

\section{Demographic Characteristics}

We first evaluated the demographic characteristics of the participant groups; these are displayed in Table 1. No significant difference in education duration was found among the four groups. However, the HC group was significantly older than the patient groups, as the patient groups generally had lower education levels than HCs of the same age. Post hoc analyses showed that the $\mathrm{HC}$ group was older than the preO group $(z=2.63, p=0.0086)$ and the postST group $(z=2.74$, $p=0.0062$ ), and the postST group was younger than the postLT group $(z=-2.15, p=0.032)$ (Table 1$)$. It is unlikely that this age difference can explain the differences in task performance reported in the following section, as previous reports have indicated that there are no effects of gender, age, or education on IGT performance (Brand et al., 2006).

\section{IGT Performance}

The aim of the IGT task is to evaluate participants' ability to learn to adjust their choice behavior based on reward feedback.

TABLE 1 | Age and education of the four subject groups.

\begin{tabular}{|c|c|c|c|c|c|c|}
\hline & $\begin{array}{c}\text { Healthy Controls } \\
\text { Mean }(S D)\end{array}$ & $\begin{array}{c}\text { Pre-operative } \\
\text { Mean (SD) }\end{array}$ & $\begin{array}{c}\text { Post-operative short term } \\
\text { Mean (SD) }\end{array}$ & $\begin{array}{c}\text { Post-operative long term } \\
\text { Mean (SD) }\end{array}$ & \multicolumn{2}{|c|}{ Welch ANOVA } \\
\hline Education (years) & $13.32(4.61)$ & $13.08(2.58)$ & $13.42(1.98)$ & $13.34(2.47)$ & 0.1453 & 0.9323 \\
\hline
\end{tabular}

There was a significant difference of age between the groups. $S D$, standard deviation. $*$ degrees of freedom $=3$.

TABLE 2 | Summary of the psychiatric rating scale scores for each patient group.

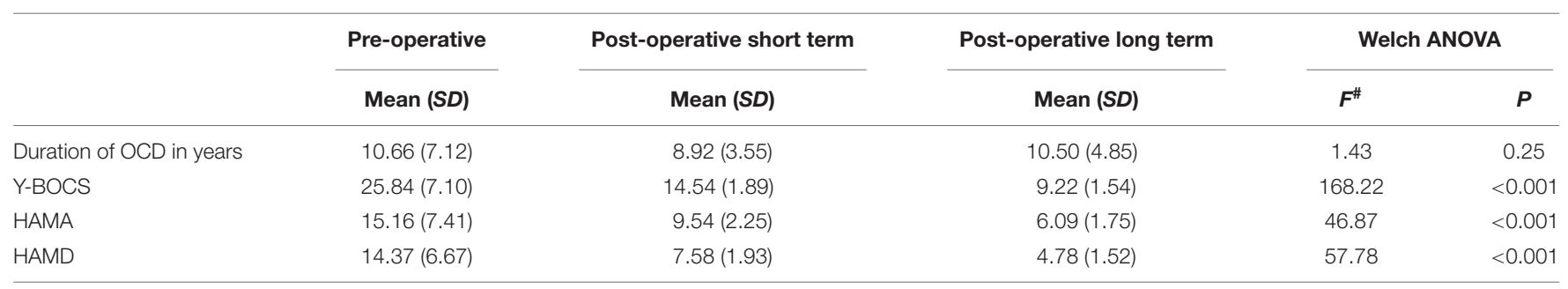

\# degree of freedom $=2$. 


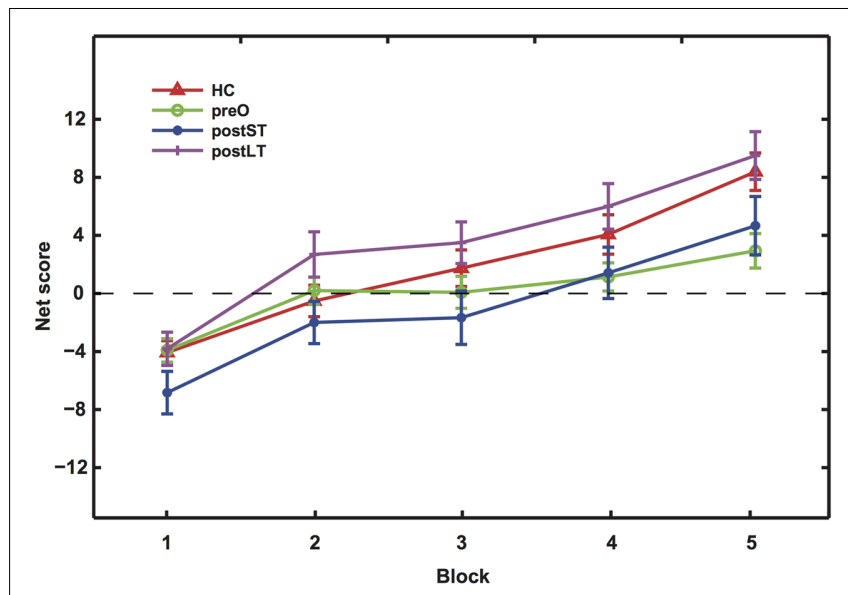

FIGURE 1 | The net scores of the five blocks during the IGT. Mean net scores for each block were calculated as $(C+D)-(A+B)$. The four curves represent the four participant groups as indicated by the legend labels. In Blocks 2 and 3 , the postLT group's scores were significantly higher than that of the postST group ( $p=0.007$ ); in Blocks 4 and 5 , both the $\mathrm{HC}$ and postLT groups achieved significantly higher scores than the pre-operative group $\left(p_{\mathrm{HC}}=0.0355 ; p_{\text {postLT }}=0.0012\right)$.

The net scores showed learning effects in all four groups, as evidenced by their increase in each progressive block (Figure 1). In the IGT, cards from the bad decks initially provide large wins. We can infer from the results that subjects in all groups picked up on this feature, as they initially chose from the bad decks more frequently. Another feature of the IGT is that once the winning cards are drawn, the remaining cards in the bad decks are more likely to result in a large loss. The large, progressive increases in HCs net scores between blocks suggest fast learning of this reversal rule regarding win probability. Interestingly, the postLT group also demonstrated score increases consistent with fast learning, exhibiting no significant difference in the net scores from those of the HC group. As a result, the $\mathrm{HC}$ and the postLT groups completed the task with higher net scores than the other two groups. Post hoc analyses were conducted to examine these changes in more detail.

Scores and statistics are summarized in Table 3. Firstly, we found a significant difference in net scores between participant groups [ANOVA, $F(3,134)=6.54, p<0.001$ ]; and a significant difference between the blocks among groups, suggesting an overall learning effect [ANOVA, $F(4,536)=43.70, p=0$ ]. There were no significant interactions between participant groups and blocks $[F(2,536)=1.30, p=0.21]$. All groups performed similarly in the first block $(p>0.1)$, suggesting that the initial learning was similar in all groups. However, their performance started to differ in intermediate Blocks 2 and $3[F(3,134)=3.66, p=0.0142]$. A follow-up $t$-test post hoc analysis with Bonferroni correction indicated that the net score of the postLT group was significantly higher than the postST group $(p<0.01)$. The net scores of the participant groups remained significantly different in Blocks 4 and $5[F(3,134)=5.66, p<0.01]$. The follow-up $t$-test with Bonferroni correction post hoc analyses showed that the net scores of both the HC and the postLT group were significantly higher than that of the pre-operative group $\left(\mathrm{p}_{\mathrm{HC}}<0.05\right.$, PpostLT $<0.01)$.

\section{IGT Choice Strategy}

To further investigate participants' choice behavior, we looked at how choice outcomes affected subjects' strategy. We hypothesized that if the subjects had learned which two decks were good, they would tend to repeat their choices even after losing points from the good decks in a particular trial. On the other hand, if they lost points after picking cards from the bad decks, they would be more likely to switch to the good decks. In contrast, if the subjects did not know which decks were good, their switch/stay behavior would be more similar to a win-stay-lose-shift strategy.

Figure 2 shows the four participant groups' tendency to repeat their choices, defined as stay probability, after wins and losses in Block 5. There was a main significant difference both among the trial conditions $[F(3,288)=4.73, p=0.0030]$ and among the participant groups $[F(3,96)=3.43, p=0.020]$ without significant interactions between the two. However, post hoc analyses revealed that subjects in both the HC group $[t(28)=4.41, p=0.00]$ and the postLT group $[t(25)=3.028, p=0.0057]$ tended to repeat their choices of the good decks regardless of whether they had just gained or lost points. In addition, their stay probability was significantly lower after they chose from the bad decks even if they had just won points. This choice pattern was not found in the subjects from the preO $[t(46)=1.79$, $p=0.083]$ and the postST groups $[t(20)=0.88, p=0.39]$. Their choices were much less consistent, indicating that they did not understand which decks were advantageous and did not adopt an appropriate strategy, even in the final block of their test session.

\section{Clinical Measures}

We evaluated subjects using three psychiatric rating scales commonly applied in the assessment of OCD patients. For the scores on all three scales, Welch ANOVA showed a main effect of operation [HAMA: $F(2,104)=46.87$, $p=0.00$; HAMD: $F(2,104)=57.78, p=0.00)$; Y-BOCS: $F(2,104)=168.22, p=0.00]$. There was a trend of decreasing scores from the pre-operative to the postST and then to the postLT patients, suggesting the operation helped alleviate both the obsessive and compulsive behaviors in these patients.

While it might be tempting to associate the observed enhanced IGT performance with the improvements in OCD symptoms, we found that there was overall a lack of correlation between the patients' psychiatric rating scale scores and their IGT performance, suggesting that the IGT and the psychiatric rating scales measured different dimensions of cognitive function. Only the HAMA scores of the postST patients showed a significant negative correlation (slope: -1.21) with their net scores (adjusted $R^{2}=0.096$, $p=0.018)$. 
TABLE 3 | Net scores of each patient group in each testing block.

\begin{tabular}{|c|c|c|c|c|c|}
\hline & Block1 Mean (SD) & Block2 Mean (SD) & Block3 Mean (SD) & Block4 Mean (SD) & Block5 Mean (SD) \\
\hline Health controls & $-4.06(4.52)$ & $-0.52(6.04)$ & $1.74(7.00)$ & $4.06(7.56)$ & $8.39(7.20)$ \\
\hline Pre-operative & $-3.92(5.75)$ & $0.20(6.62)$ & $0.08(7.86)$ & $1.13(6.93)$ & $2.94(8.48)$ \\
\hline Post-operative short term & $-6.83(7.20)$ & $-2.00(7.10)$ & $-1.67(9.02)$ & $1.42(8.68)$ & $4.67(9.85)$ \\
\hline Post-operative long term & $-3.81(6.47)$ & $2.69(8.81)$ & $3.5(8.11)$ & $6.00(8.90)$ & $9.50(9.30)$ \\
\hline $\mathrm{F}$ & 1.58 & \multicolumn{2}{|c|}{3.66} & \multicolumn{2}{|c|}{5.66} \\
\hline$p$ & 0.20 & \multicolumn{2}{|c|}{0.014} & \multicolumn{2}{|c|}{0.0011} \\
\hline
\end{tabular}

Standard deviations are shown in the parentheses. The F and p-values are from the ANOVA tests of the group effects.

\section{DISCUSSION}

The results of the present study converge with extensive research in the field of OCD demonstrating impaired decision making associated with the disorder, particularly in refractory OCD patients, and further suggest that $\mathrm{AC}$ results in improvement across obsessive-compulsive, depressive and anxious clinical symptoms as well as decision-making ability under ambiguity. Our findings indicate that improved decision making occurs over time as a result of the normalization of aberrant CSTC activity, rather than as an instantaneous result of the neurosurgical lesion, therefore possibly constituting further evidence for the role of the CSTC circuitry in decision-making ability. We found no evidence for an association between clinical symptoms and decision making, suggesting that different neural substrates are at play.

Anatomically, the prefrontal cortex (PFC) is essential for the neural processes that underlie decision making and its sub processes, including planning, inductive reasoning, reward processing and manipulating complex information (Cservenka and Nagel, 2012; Rebola et al., 2012). Studies often differentiate three sub-regions of the PFC: the dorsolateral prefrontal cortex (DLPFC), the orbitofrontal cortex (OFC) and the anterior cingulate cortex (ACC) (Fuster, 2001). The OFC occupies the ventral region of the $\mathrm{PFC}$ and can further be divided into the ventromedial and ventrolateral portions, with the ventromedial

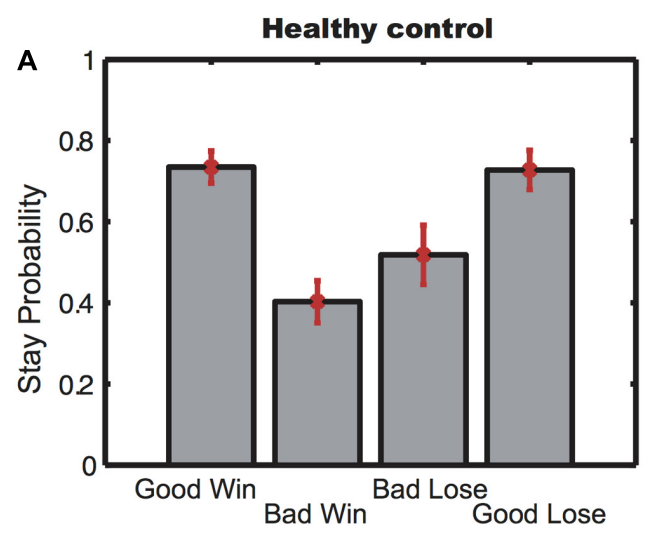

B

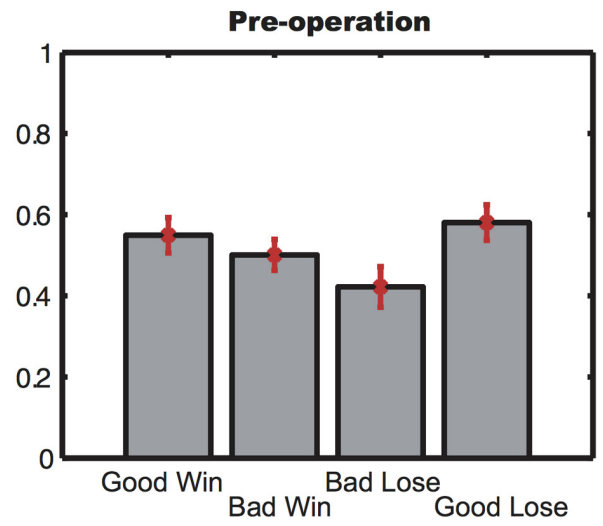

Post-operation short term

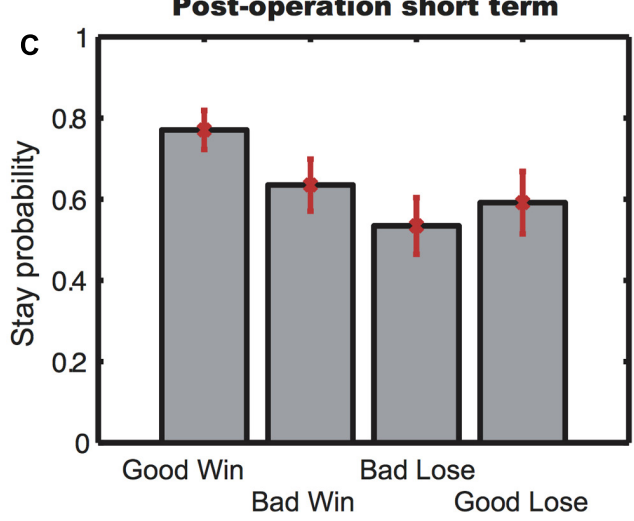

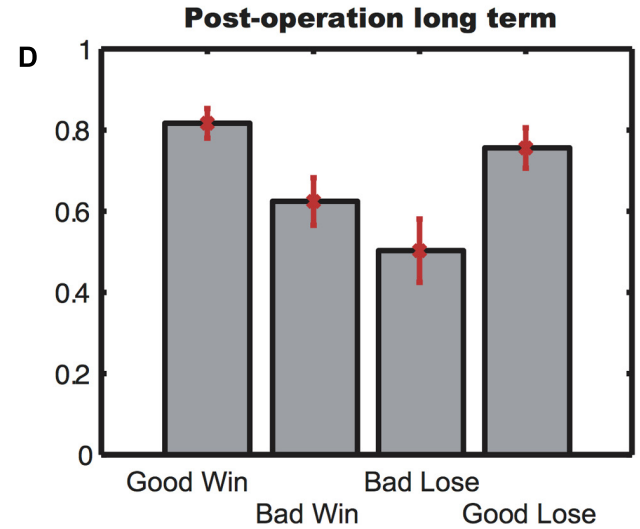

Bad Win Good Lose

FIGURE 2 | The stay probability of Block 5. (A) Stay probability for the healthy controls; (B) stay probability for the pre-operative group; (C) stay probability for the postST group; (D) stay probability for the postLT group. Error bars indicate SEM. 
OFC most frequently implicated in OCD (Bechara et al., 1994). This area appears critical in integrating affective information relayed from the other limbic areas and directly signaling certain reward types. In environments where rewarding options may constantly change, the OFC plays an important role in adaptive behavior (Elliott et al., 2000). It maintains a representation of reward history and modifies former reward representations that may have become disadvantageous. Together with frequently reported neuroimaging findings of aberrant OFC activity in OCD patients, these lines of evidence lend support to an association between reward mechanisms and symptomatology of the disorder. OFC impairments are often acknowledged as the most supported account for the inadequate behavioral choices and inflexibility of behavioral change typically expressed in OCD, although the underlying circuitry is likely far more complex.

Interestingly, patients with lesions in the OFC show deficits in their IGT performance similar to OCD patients (Bechara et al., 1994). More generally, recent research has shown improvements in verbal memory, visual memory, visuospatial skills and executive function in treatment-refractory $\mathrm{OCD}$ following $\mathrm{AC}$ as compared to both a non-surgical treatment group and healthy controls (Gong et al., 2017). Our study therefore provides further evidence that abnormal OFC functionality may be a contributing factor of the OCD behavior. We postulate that AC may enhance OFC activity via its disinhibition, though the mechanism by which this occurs is not immediately obvious. One of the major pathways in the anterior internal capsule carries projections from the thalamus to the frontal cortex. However, these projections are excitatory. Corticopontine projections also pass through the internal capsule, although they are unlikely to affect the OFC. It is therefore likely that capsulotomy works by disrupting the basal ganglia's internal connections between the caudate and lentiform nucleus. Modifying these connections may affect the output of the basal ganglia, leading to the disinhibition of the OFC. This indirect modulation could also explain why behavioral improvement is mostly observed only in the long run (Pepper et al., 2015).

We found that the AC improved OCD symptoms as indicated by clinical scores, which is consistent with previous reports (D'Astous et al., 2013). In addition, our observed outcome of improved decision-making ability occurring over time independently of symptom alleviation is in line with that of Csigo et al. (2010), and is indicative of possible differences in the neural substrates underlying clinical symptoms and neuropsychological difficulties in OCD. The objective of neurosurgical techniques is to influence the connections between cortical areas (e.g., orbitofrontal cortex and cingulum), the basal ganglia (particularly the caudate nucleus) and the medial dorsal thalamic nucleus. Our findings indicate that IGT performance is not directly influenced by the instantaneous changes caused by the disruption of these connections. The critical role of receptor function in the neural circuitry mediating behavioral control evokes the possibility that decision making as measured by the IGT task is modulated by dopamine or serotonin signaling. Neurochemical alterations of the serotonergic and dopamine systems are assumed to be involved in the mediation of OCD symptoms (Denys et al., 2004). Serotonin (5-HT) has received the most attention in OCD, as enhancement of 5-HT neurotransmission results in a therapeutic effect; potent 5-HT re-uptake inhibitors are effective treatments for OCD (Huang and Williams, 2007). However, there is evidence that dopamine receptor levels modulate impulsivity and effortful decision making in mice (Simon et al., 2013). Furthermore, striatal dopamine D2/D3-receptor availability was demonstrated to have an inverse relationship with response inhibition speed on the Stop-Signal Task (a measure of impulsive behavior) and a positive relationship with inhibition-related fMRI activation in the fronto-striatal neural circuitry in healthy individuals (Ghahremani et al., 2012). Further research is needed to determine whether long-term rebalancing of neurotransmitter levels after AC contributes to changes in decision-making ability and other cognitive facets, such as impulsivity, that are commonly impaired in OCD.

Various accounts consider neuropsychological impairment an intermediate phenomenon linking aberrant brain activity with clinical symptomatology (Zhang et al., 2015). Moreover, unaffected relatives of individuals with OCD consistently exhibit comparable deficits in decision making to probands (Viswanath et al., 2009; Cavedini et al., 2010). There is evidence that behavioral impairment in both is associated with gray matter abnormalities in regions including the orbitofrontal cortex, indicating a heritable component (Menzies et al., 2007). However, it remains unclear whether this component is a primary feature of disease circuitry or a compensatory mechanism for other primary features. Future studies aimed at improved characterization of the neural substrates of neuropsychological and clinical characteristics before and after surgical intervention can help to elucidate the complex overlap and divergence between these disease characteristics.

Finally, our sample was comprised of individuals with severe OCD that is resistant to conventional treatment methods. Studies have indicated that individuals with refractory OCD exhibit a higher degree of neuropsychological deficits than treatment responsive individuals in terms of attention, set-shifting ability, linguistic performance, decision making, impulsivity (Csigo et al., 2010). Interestingly, there is also evidence that poor performance on the IGT is predictive of poor pharmacological treatment outcomes (Cavedini et al., 2002). However, it is important to bear in mind that dysfunction within any of the other neural systems that feed into the OFC/vmPFC system can lead to similar decision-making impairments, particularly when the disorders are marked by recognized heterogeneity in neural and symptomatological manifestation.

\section{CONCLUSION}

The current study corroborates previous findings supporting the effectiveness of $\mathrm{AC}$ for the treatment of refractory OCD. Our results revealed that long-term changes after $\mathrm{AC}$ in the brain circuit may be revealed by the patients' improvements in IGT. 
These changes were not correlated to the psychiatric rating scales, which started to show improvements in the patients shortly after AC. Future studies will be needed to demonstrate the neural circuitry underlying both categories of change and how they are affected by AC across time.

\section{AUTHOR CONTRIBUTIONS}

TY, BS, and SZ conceived of the project. TY and YC designed and supervised the experiments. YC and TY analyzed the data. CZ, ST, KZ, HJ, HG, YX, TW, BS, and SZ collected the data. CZ, ST, TW, BS, and SZ performed the surgery. $\mathrm{CZ}, \mathrm{YC}, \mathrm{KZ}$, and TY wrote the paper with inputs from all authors.

\section{REFERENCES}

Abramowitz, J. S., Taylor, S., and McKay, D. (2009). Obsessive-compulsive disorder. Lancet 374, 491-499. doi: 10.1016/S0140-6736(09)60240-3

Bechara, A., Damasio, A. R., Damasio, H., and Anderson, S. W. (1994). Insensitivity to future consequences following damage to human prefrontal cortex. Cognition 50, 7-15. doi: 10.1016/0010-0277(94)90018-3

Bechara, A., Damasio, H., Tranel, D., and Anderson, S. W. (1998). Dissociation of working memory from decision making within the human prefrontal cortex. J. Neurosci. 18, 428-437.

Bechara, A., Dolan, S., Denburg, N., Hindes, A., Anderson, S. W., and Nathan, P. E. (2001). Decision making deficits, linked to a dysfunctional ventromedial prefrontal cortex, revealed in alcohol and stimulant abusers. Neuropsychologia 39, 376-389. doi: 10.1016/S0028-3932(00)00136-6

Bechara, A., Tranel, D., and Damasio, H. (2000). Characterization of the decisionmaking deficit of patients with ventromedial prefrontal cortex lesions. Brain 123(Pt 1), 2189-2202. doi: 10.1093/brain/123.11.2189

Bloch, M. H., Landeros-Weisenberger, A., Kelmendi, B., Coric, V., Bracken, M. B., and Leckman, J. F. (2006). A systematic review: antipsychotic augmentation with treatment refractory obsessive-compulsive disorder. Mol. Psychiatry 11, 622-632. doi: 10.1038/sj.mp.4001823

Bloch, M. H., Wasylink, S., Landeros-Weisenberger, A., Panza, K. E., Billingslea, E., Leckman, J. F., et al. (2012). Effects of ketamine in treatment-refractory obsessive-compulsive disorder. Biol. Psychiatry 72, 964-970. doi: 10.1016/j. biopsych.2012.05.028

Brand, M., Labudda, K., and Markowitsch, H. J. (2006). Neuropsychological correlates of decision-making in ambiguous and risky situations. Neural Netw. 19, 1266-1276. doi: 10.1016/j.neunet.2006.03.001

Brown, L. T., Mikell, C. B., Youngerman, B. E., Zhang, Y., McKhann, G. M., and Sheth, S. A. (2016). Dorsal anterior cingulotomy and anterior capsulotomy for severe, refractory obsessive-compulsive disorder: a systematic review of observational studies. J. Neurosurg. 124, 77-89. doi: 10.3171/2015.1.JNS14681

Cavallaro, R., Cavedini, P., Mistretta, P., Bassi, T., Angelone, S. M., Ubbiali, A., et al. (2003). Basal-corticofrontal circuits in schizophrenia and obsessive-compulsive disorder: a controlled, double dissociation study. Biol. Psychiatry 54, 437-443. doi: 10.1016/S0006-3223(02)01814-0

Cavedini, P., Riboldi, G., D’Annucci, A., Belotti, P., Cisima, M., and Bellodi, L. (2002). Decision-making heterogeneity in obsessive-compulsive disorder: ventromedial prefrontal cortex function predicts different treatment outcomes. Neuropsychologia 40, 205-211. doi: 10.1016/S0028-3932(01)00077-X

Cavedini, P., Zorzi, C., Piccinni, M., Cavallini, M. C., and Bellodi, L. (2010). Executive dysfunctions in obsessive-compulsive patients and unaffected relatives: searching for a new intermediate phenotype. Biol. Psychiatry 67, 1178-1184. doi: 10.1016/j.biopsych.2010.02.012

Cservenka, A., and Nagel, B. J. (2012). Risky decision-making: an fMRI study of youth at high risk for alcoholism. Alcohol. Clin. Exp. Res. 36, 604-615. doi: 10.1111/j.1530-0277.2011.01650.x

\section{FUNDING}

The SHSMU-ION Research Center for Brain Disorders; National Natural Science Foundation of China Grant (81771482) to BS; Chinese Academy of Sciences Hundreds of Talents Program and Science and Technology Commission of Shanghai Municipality (15JC1400104) to TY.

\section{ACKNOWLEDGMENTS}

We thank Dr. Zhengdao Deng (Department of Functional Neurosurgery, Ruijin Hospital) and Yuehan Wei, Siyi Qi, Yue Zhou, and Gulimiheranmu Maisumu from Shanghai Jiaotong University School of Medicine for their help in collecting data.

Csigo, K., Harsanyi, A., Demeter, G., Rajkai, C., Nemeth, A., and Racsmany, M. (2010). Long-term follow-up of patients with obsessive-compulsive disorder treated by anterior capsulotomy: a neuropsychological study. J. Affect. Disord. 126, 198-205. doi: 10.1016/j.jad.2010.02.127

D'Astous, M., Cottin, S., Roy, M., Picard, C., and Cantin, L. (2013). Bilateral stereotactic anterior capsulotomy for obsessive-compulsive disorder: long-term follow-up. J. Neurol. Neurosurg. Psychiatry 84, 1208-1213. doi: 10.1136/jnnp2012-303826

Denys, D., van der Wee, N., Janssen, J., De Geus, F., and Westenberg, H. G. (2004). Low level of dopaminergic D2 receptor binding in obsessive-compulsive disorder. Biol. Psychiatry 55, 1041-1045. doi: 10.1016/j.biopsych.2004. 01.023

Elliott, R., Dolan, R. J., and Frith, C. D. (2000). Dissociable functions in the medial and lateral orbitofrontal cortex: evidence from human neuroimaging studies. Cereb. Cortex 10, 308-317. doi: 10.1093/cercor/10.3.308

Fineberg, N. A., Chamberlain, S. R., Hollander, E., Boulougouris, V., and Robbins, T. W. (2011). Translational approaches to obsessive-compulsive disorder: from animal models to clinical treatment. Br. J. Pharmacol. 164, 1044-1061. doi: 10.1111/j.1476-5381.2011.01422.x

Fuster, J. M. (2001). The prefrontal cortex - an update: time is of the essence. Neuron 30, 319-333. doi: 10.1016/S0896-6273(01)00285-9

Ghahremani, D. G., Lee, B., Robertson, C. L., Tabibnia, G., Morgan, A. T., De Shetler, N., et al. (2012). Striatal dopamine $\mathrm{D}_{2} / \mathrm{D}_{3}$ receptors mediate response inhibition and related activity in frontostriatal neural circuitry in humans. J. Neurosci. 32, 7316-7324. doi: 10.1523/JNEUROSCI.4284-11.2012

Gong, F., Li, P., Li, B., Zhang, S., Zhang, X., Yang, S., et al. (2017). A study of cognitive function in treatment-refractory obsessive-compulsive disorder treated with capsulotomy. J. Neurosurg. doi: 10.3171/2016.9.JNS152494 [Epub ahead of print].

Goodman, W. K., Price, L. H., Rasmussen, S. A., Mazure, C., Fleischmann, R. L., Hill, C. L., et al. (1989). The yale-brown obsessive compulsive scale. I. Development, use, and reliability. Arch. Gen. Psychiatry 46, 1006-1011. doi: 10.1001/archpsyc.1989.01810110048007

Grant, J. E. (2014). Clinical practice: obsessive-compulsive disorder. N. Engl. J. Med. 371, 646-653. doi: 10.1056/NEJMcp1402176

Greenberg, B. D., Rauch, S. L., and Haber, S. N. (2010). Invasive circuitry-based neurotherapeutics: stereotactic ablation and deep brain stimulation for OCD. Neuropsychopharmacology 35, 317-336. doi: 10.1038/npp.2009.128

Hamilton, M. (1986). “The Hamilton rating scale for depression," in Assessment of Depression, eds N. Sartorius and T. A. Ban (Berlin: Springer), 143-152.

Huang, Y., and Williams, W. A. (2007). Enhanced selective serotonin re-uptake inhibitors as antidepressants: 2004-2006. Expert Opin. Ther. Pat. 17, 889-907. doi: $10.1517 / 13543776.17 .8 .889$

Jung, H. H., Kim, S. J., Roh, D., Chang, J. G., Chang, W. S., Kweon, E. J., et al. (2014). Bilateral thermal capsulotomy with MR-guided focused ultrasound for patients with treatment-refractory obsessive-compulsive disorder: a proof-of-concept study. Mol. Psychiatry 20, 1205-1211. doi: 10.1038/mp.2014.154 
Lawrence, N. S., Wooderson, S., Mataix-Cols, D., David, R., Speckens, A., and Phillips, M. L. (2006). Decision making and set shifting impairments are associated with distinct symptom dimensions in obsessive-compulsive disorder. Neuropsychology 20, 409-419. doi: 10.1037/0894-4105.20.4.409

Liu, K., Zhang, H., Liu, C., Guan, Y., Lang, L., Cheng, Y., et al. (2008). Stereotactic treatment of refractory obsessive compulsive disorder by bilateral capsulotomy with 3 years follow-up. J. Clin. Neurosci. 15, 622-629. doi: 10.1016/j.jocn.2007. 07.086

Maier, W., Buller, R., Philipp, M., and Heuser, I. (1988). The Hamilton Anxiety Scale: reliability, validity and sensitivity to change in anxiety and depressive disorders. J. Affect. Disord. 14, 61-68. doi: 10.1016/0165-0327(88)90072-9

Mallet, L., Polosan, M., Jaafari, N., Baup, N., Welter, M.-L., Fontaine, D., et al. (2008). Subthalamic nucleus stimulation in severe obsessive-compulsive disorder. N. Engl. J. Med. 359, 2121-2134. doi: 10.1056/NEJMoa0708514

Menzies, L., Achard, S., Chamberlain, S. R., Fineberg, N., Chen, C.-H., Del Campo, N., et al. (2007). Neurocognitive endophenotypes of obsessivecompulsive disorder. Brain 130, 3223-3236. doi: 10.1093/brain/awm205

Milad, M. R., and Rauch, S. L. (2012). Obsessive-compulsive disorder: beyond segregated cortico-striatal pathways. Trends Cogn. Sci. 16, 43-51. doi: 10.1016/ j.tics.2011.11.003

Nakao, T., Okada, K., and Kanba, S. (2014). Neurobiological model of obsessive-compulsive disorder: evidence from recent neuropsychological and neuroimaging findings. Psychiatry Clin. Neurosci. 68, 587-605. doi: 10.1111/ pcn. 12195

Nielen, M. M. A., Veltman, D. J., De Jong, R., Mulder, G., and Den Boer, J. A. (2002). Decision making performance in obsessive compulsive disorder. J. Affect. Disord. 69, 257-260. doi: 10.1016/S0165-0327(00)00381-5

Norman, L. J., Carlisi, C., Lukito, S., Hart, H., Mataix-Cols, D., Radua, J., et al. (2016). Structural and functional brain abnormalities in attention-deficit/hyperactivity disorder and obsessive-compulsive disorder: a comparative meta-analysis. JAMA Psychiatry 73, 815-825. doi: 10.1001/jamapsychiatry.2016.0700

Nuttin, B., Wu, H., Mayberg, H., Hariz, M., Gabriëls, L., Galert, T., et al. (2014). Consensus on guidelines for stereotactic neurosurgery for psychiatric disorders. J. Neurol. Neurosurg. Psychiatry 85, 1003-1008. doi: 10.1136/jnnp-2013-306580

Odlaug, B. L., Weinhandl, E., Mancebo, M. C., Mortensen, E. L., Eisen, J. L., Rasmussen, S. A., et al. (2014). Excluding the typical patient: thirty years of pharmacotherapy efficacy trials for obsessive-compulsive disorder. Annu. Clin. Psychiatry 26, 39-46.

Pauls, D. L., Abramovitch, A., Rauch, S. L., and Geller, D. A. (2014). Obsessivecompulsive disorder: an integrative genetic and neurobiological perspective. Nat. Rev. Neurosci. 15, 410-424. doi: 10.1038/nrn3746

Pepper, J., Hariz, M., and Zrinzo, L. (2015). Deep brain stimulation versus anterior capsulotomy for obsessive-compulsive disorder: a review of the literature. J. Neurosurg. 122, 1028-1037. doi: 10.3171/2014.11.JNS132618

Rebola, J., Castelhano, J., Ferreira, C., and Castelo-Branco, M. (2012). Functional parcellation of the operculo-insular cortex in perceptual decision making: an fMRI study. Neuropsychologia 50, 3693-3701. doi: 10.1016/j.neuropsychologia. 2012.06.020
Saxena, S., Bota, R. G., and Brody, A. L. (2001). Brain-behavior relationships in obsessive-compulsive disorder. Semin. Clin. Neuropsychiatry 6, 82-101. doi: $10.1053 /$ scnp.2001.21833

Schlösser, R. G. M., Wagner, G., Schachtzabel, C., Peikert, G., Koch, K., Reichenbach, J. R., et al. (2010). Fronto-cingulate effective connectivity in obsessive compulsive disorder: a study with fMRI and dynamic causal modeling. Hum. Brain Mapp. 31, 1834-1850. doi: 10.1002/hbm. 20980

Sheehan, D. V., Lecrubier, Y., Sheehan, K. H., Amorim, P., Janavs, J., Weiller, E., et al. (1998). The Mini-International Neuropsychiatric Interview (M.I.N.I.): the development and validation of a structured diagnostic psychiatric interview for DSM-IV and ICD-10. J. Clin. Psychiatry 59(Suppl. 20):22-33;quiz 34-57. doi: 10.1016/S0924-9338(99)80239-9

Simon, N. W., Beas, B. S., Montgomery, K. S., Haberman, R. P., Bizon, J. L., and Setlow, B. (2013). Prefrontal cortical-striatal dopamine receptor mRNA expression predicts distinct forms of impulsivity. Eur. J. Neurosci. 37, 1779-1788. doi: 10.1111/ejn.12191

Viswanath, B., Janardhan Reddy, Y. C., Kumar, K. J., Kandavel, T., and Chandrashekar, C. R. (2009). Cognitive endophenotypes in OCD: a study of unaffected siblings of probands with familial OCD. Prog Neuropsychopharmacol. Biol. Psychiatry 33, 610-615. doi: 10.1016/j.pnpbp. 2009.02.018

Whiteside, S. P., Port, J. D., and Abramowitz, J. S. (2004). A meta-analysis of functional neuroimaging in obsessive-compulsive disorder. Psychiatry Res. 132, 69-79. doi: 10.1016/j.pscychresns.2004.07.001

Zhan, S., Wei, L., Li, D., Pan, S., Pan, Y., Li, Y., et al. (2014). Long-term follow-up of bilateral anterior capsulotomy in patients with refractory obsessive-compulsive disorder. Clin. Neurol. Neurosurg. 119, 91-95. doi: 10.1016/j.clineuro.2014. 01.009

Zhang, L., Dong, Y., Ji, Y., Tao, R., Chen, X., Ye, J., et al. (2015). Trait-related decision making impairment in obsessive-compulsive disorder: evidence from decision making under ambiguity but not decision making under risk. Sci. Rep. 5:17312. doi: 10.1038/srep17312

Zuo, C., Ma, Y., Sun, B., Peng, S., Zhang, H., Eidelberg, D., et al. (2013). Metabolic imaging of bilateral anterior capsulotomy in refractory obsessive compulsive disorder: an FDG PET study. J. Cereb. Blood Flow Metab. 33, 880-887. doi: $10.1038 /$ jcbfm.2013.23

Conflict of Interest Statement: The authors declare that the research was conducted in the absence of any commercial or financial relationships that could be construed as a potential conflict of interest.

Copyright (c) 2017 Zhang, Chen, Tian, Wang, Xie, Jin, Lin, Gong, Zeljic, Sun, Yang and Zhan. This is an open-access article distributed under the terms of the Creative Commons Attribution License (CC BY). The use, distribution or reproduction in other forums is permitted, provided the original author(s) or licensor are credited and that the original publication in this journal is cited, in accordance with accepted academic practice. No use, distribution or reproduction is permitted which does not comply with these terms. 